\title{
Subtalar versus triple arthrodesis after intra-articular calcaneal fractures
}

\author{
Tim Schepers • Brenda C. T. Kieboom • \\ Gert H. J. M. Bessems • Lucas M. M. Vogels • \\ Esther M. M. van Lieshout • Peter Patka
}

Received: 18 April 2009/Accepted: 18 February 2010/Published online: 23 March 2010

(C) Springer-Verlag 2010

\begin{abstract}
Depending upon initial treatment, between 2 and $30 \%$ of patients with a displaced intra-articular calcaneal fracture require a secondary arthrodesis. The aim of this study was to investigate the effect of subtalar versus triple arthrodesis on functional outcome. A total of 33 patients with 37 secondary arthrodeses (17 subtalar and 20 triple) with a median follow-up of 116 months were asked to complete questionnaires regarding disease-specific functional outcome (Maryland Foot Score, MFS), quality of life (SF-36) and overall satisfaction with the treatment (Visual Analogue Scale, VAS). Patient groups were comparable considering median age at fracture, initial treatment (conservative or operative), time to arthrodesis, median follow-up, and post-arthrodesis radiographic angles. The MFS score was similar after subtalar versus triple arthrodesis (59 vs. 56 points; $P=0.79$ ). No statistically significant difference was found for the SF-36 (84 vs. 83 points; $P=0.67$ ) and the VAS (5 vs. $6 ; P=0.21$ ). Smoking was statistically significantly associated with a non-union $\left(\chi^{2}=6.60, P=0.017\right)$. The current study suggests that there is no significant difference in functional outcome between an in situ subtalar or triple arthrodesis as a salvage technique for symptomatic arthrosis after an intra-articular calcaneal fracture. Smoking is a risk factor for non-union.
\end{abstract}

T. Schepers $(\varangle)$ - B. C. T. Kieboom - L. M. M. Vogels ·

E. M. M. van Lieshout · P. Patka

Department of Surgery-Traumatology, Erasmus MC,

University Medical Centre Rotterdam, Room H-822k,

P.O. Box 2040, 3000 CA Rotterdam, The Netherlands

e-mail: t.schepers@erasmusmc.nl

G. H. J. M. Bessems

Department of Orthopaedic Surgery, Erasmus MC,

University Medical Centre Rotterdam,

Rotterdam, The Netherlands
Keywords Calcaneus - Arthrodesis · Outcome

\section{Introduction}

There is a considerable body of literature on the treatment of displaced intra-articular calcaneal fractures. Although open reduction and internal fixation (ORIF) is currently used by most surgeons, approximately half of all patients are managed conservatively or percutaneously [1]. Assessing the outcome by means of quality of life scores such as Short-Form-36 (SF-36) or visual analogue scale (VAS) revealed no apparent benefit in functional outcome of operative treatment over conservative treatment [2]. However, the need for a secondary arthrodesis is approximately $21-30 \%$ after initial conservative treatment versus $2-5 \%$ after operative treatment [2-4]. Currently, there is insufficient evidence whether patients requiring a late subtalar arthrodesis after initial operative treatment do better than those initially treated conservatively [5].

The late sequelae after an intra-articular calcaneal fracture have been well described and include incongruence and painful arthritis of the posterior talocalcaneal and calcaneo-cuboid joints, arthrofibrosis with a stiff subtalar joint, lateral impingement of the peroneal tendons and fibular abutment, impaired dorsiflexion of the ankle joint and tibio-talar impingement, broadening of the calcaneus with loss of height, hindfoot varus or valgus deformity, subluxation of the Chopart joint, pes planus, neuropathies, and a painful heel pad $[4,6,7]$. The predicament of a painful old calcaneal fracture [8] has encouraged the development of different salvage procedures. These are based upon the four main indications for an arthrodesis: (1) to achieve correction of the deformity, (2) relieve pain, (3) stabilise the joints, and (4) improve functional outcome 
[9-12]. Discussion remains whether these deformities should be managed by subtalar in situ, triple, or bone-block distraction arthrodesis. It also remains unclear whether a secondary arthrodesis will result in improved outcome after initial operative treatment, compared with initial conservative treatment [5].

The objective of this study was to compare the outcome as measured by disease-specific outcome measures and generic quality of life scores after either secondary in situ subtalar or triple arthrodesis for painful post-traumatic arthritis and malunion following an intra-articular calcaneal fracture, which may have been treated initially either conservatively or operatively.

\section{Patients and methods}

At our institution, trauma and orthopaedic surgery are separate specialities, and surgeons from both provide the fracture care in trauma patients. Initial treatment for calcaneal fractures by the trauma surgeons tends to be operative (percutaneous or ORIF), whereas orthopaedic surgeons generally prefer conservative treatment. Our hospital acts as a tertiary referral centre for complex cases. Conservative and ORIF methods are established treatment modalities and are performed following standard procedures, as described in the literature [2, 3, 13]. ORIF is performed mainly outside our referring hospital, through the extended lateral approach as advised by the AO. Fixation is with various types of plates and screws, and no external fixation is utilised. The percutaneous treatment follows the technique described by Forgon and Zadravecz, with modifications previously described from this unit [14]. Briefly, this method relies on distraction of the fracture with realignment of the hindfoot, restoration of height, width, varus/valgus malalignment, and gross incongruence at the subtalar joint, utilising ligamentotaxis and subsequent percutaneous screw fixation [14].

The decision to perform a subtalar or triple arthrodesis as salvage procedure is based upon patient history, physical examination, pre-operative radiographs, and surgeon preference. Several patients were referred after various different treatment modalities or failed subtalar arthrodesis after an intra-articular calcaneal fracture.

\section{Patient selection}

All electronic operation records between 1990 and 2005 were searched for subtalar or triple arthrodesis using the appropriate operation codes. Three hundred and fifty-four records were identified and searched manually for the operative indications, to select the patients with an arthrodesis performed for the painful sequelae of an intra-articular calcaneal fracture. Excluded were 150 congenital deformities (e.g. clubfeet), 57 patients with rheumatic changes, and 91 patients with secondary arthrosis not caused by a calcaneal fracture (e.g. talar fractures, subtalar dislocation, posterior tibialis tendon dysfunction). A minimum follow-up of 2 years was specified, providing sufficient time for the arthrodesis to heal and for patients to adapt to the new situation. All patients had suffered from persisting pain and restrictions in daily activities, not relieved with conservative measures (NSAID and shoe modifications), and had a positive, pain-relieving effect of a lidocaine injection into the subtalar joint.

\section{Operative technique}

In all subtalar and triple arthrodeses, a lateral approach was used in one of two ways: a curved Ollier incision over the sinus tarsi or a longitudinal incision starting $1 \mathrm{~cm}$ caudal to the tip of the distal fibula towards the base of the fourth metatarsal. An additional medial incision over the talonavicular joint was used on four occasions when a triple arthrodesis was performed. The technique used for the in situ fusion has been described previously [15-17]. The need for bone grafting and internal fixation was determined during operation. Decompression of the lateral calcaneal wall was performed where lateral impingement was evident after realignment of the hindfoot $(N=3)$ [18]. Follow-up treatment consisted of 4-6 weeks in a non-weight bearing below-knee cast followed by 4-6 weeks weight bearing in a below-knee cast.

\section{Non-union and complications}

The numbers of infections and non-unions at the arthrodesis site was determined by reviewing the charts and radiographs of all patients.

\section{Radiological evaluation}

Böhlers and Gissane's angle were determined from the pre-operative radiographs of all patients. In the pre- and post-arthrodesis radiographs, the following angles were measured as previously described [19]: calcaneal inclination, talar declination, and talocalcaneal angle. Prearthrodesis CT scans or axial radiographs were classified as described by Stephens and Sanders [20, 21]. This classification describes three types of malunion: in type 1 there is bulging of the lateral wall without subtalar arthrosis; type 2 shows lateral wall exostosis and an incongruent joint with subtalar arthrosis, without varus malalignment; in type 3 there is lateral wall exostosis, subtalar arthrosis and more than 10 degrees of hindfoot varus [21]. 
Clinical evaluation

The study was approved by the local Medical Ethics Committee. Clinical evaluation was by postal questionnaire and reviewing all the patient records. All eligible patients were sent a set of questionnaires, which included the SF36, a single question VAS and the Maryland Foot Score (MFS) [22]. The MFS comprises 100 possible points and allows for subjective assessment of the foot and ankle with respect to pain, cosmesis, need for shoe modifications, range of motion and general function, the latter including walking distance, stability, need for support, limping, climbing stairs, and difficulties on different terrains. The MFS has previously been shown to be a reliable and valid indicator for determining outcome after intra-articular calcaneal fractures [23]. The SF-36 consists of 36 items that assess health-related quality of life by means of eight health domains: physical functioning $(\mathrm{PF})$; role limitations due to physical health (RP); bodily pain (BP); social functioning (SF); vitality energy or fatigue (VT); general health perceptions $(\mathrm{GH})$; role limitations due to emotional problems (RE); and general mental health $(\mathrm{MH})$. The PF, $\mathrm{RP}$, and BP scales reflect the physical elements of health (PCS); the SF, RE, and MH represent psychological aspects; and VT and GH indicate the subjective perception of health. Scores ranging from 0 to 100 points are derived for each domain, with lower scores indicating poorer function. These scores were converted to a norm-based score and compared with the norms for the general population of the United States (1998). In the US population, each scale was scored to have the same average (50 points) and the same standard deviation (10 points). Calculating norm-based scores using the Dutch and US populations provides similar results for the eight health domains. However, the weighing factors for calculating PCS and MCS for the Dutch population were not available; therefore, the US population was used as reference.

In addition to the retrieved data from the medical charts, patients were asked for their weight and height (from this data the BMI was calculated) and smoking behaviour as these might affect union rates and outcome.

\section{Statistical analysis}

Statistical analysis was performed using the Statistical Package for the Social Sciences (SPSS) version 12.0.1 (SPSS, Chicago, IL, USA). Functional outcome after subtalar arthrodesis versus triple arthrodesis was assessed using the non-parametric Mann-Whitney U test. In addition, the influence of smoking on the rate of non-unions was investigated using a Chi-square analysis. Associations were considered statistically significant with a $P$-value of $<0.05$.

\section{Results}

A total of 56 patients with in total 61 arthrodeses, either in situ subtalar or in situ triple, were identified during the first screening. Fourteen patients were excluded, as they had moved and their current address could not be retrieved. Questionnaires were sent to the remaining 42 patients of whom 33 responded and were used for the analysis. The median age at injury was 39 years (range 19-74), with 23 male and 10 female. In these patients 37 arthrodeses were performed (16 right sided, 13 left sided, and four bilateral). Twenty-four fractures were initially treated conservatively, and 13 patients operatively (six ORIF, seven percutaneous). The median time between injury and arthrodesis was 29 months (range 9-456). There were 27 subtalar and ten triple arthrodeses performed as the initial salvage procedure. Ten patients, initially treated with a subtalar arthrodesis, were completed to a triple arthrodesis, seven because of a non-union at the subtalar joint and progressive arthrosis at the Chopart joint, and in three because of progressive arthrosis. Thus, at the time of follow-up there were 17 subtalar and 20 triple arthrodeses.

An in situ arthrodesis was performed in all cases, and no bone-block distraction or sliding-osteotomy techniques were used. In 19 cases no internal fixation was used, whereas others were internally stabilised using staples $(N=9)$ or cannulated screws $(N=9)$. Bone graft was harvested from the iliac crest in eight cases, from the proximal tibia in nine and from local sources in 8; in 12 cases no bone graft was used.

\section{Pseudarthrosis and complications}

A non-union was seen in one or more joints in 14 feet. In one out of 10 initial triple arthrodeses, a non-union occurred of the talonavicular and talocalcaneal joints. In 13 of 27 initial subtalar arthrodeses, a non-union of the talocalcaneal joint occurred. In the total population of 56 patients with 61 arthrodeses, a total of 18 non-unions occurred (30\%). Revision surgery was performed in these cases, and three of these underwent a second revision surgery due to a persistent non-union. Three superficial infections and one osteomyelitis were seen in four patients, and screws or staples removed in nine.

\section{Radiological results}

Median pre-operative Böhlers and Gissanes angle for the entire group was 5 degrees $\left(\mathrm{P}_{25}-\mathrm{P}_{75}, 15-17\right)$ and 112 degrees $\left(\mathrm{P}_{25}-\mathrm{P}_{75}, 105-125\right)$, respectively. Pre-operative talocalcaneal, calcaneal inclination, and talar declination angles were 19 (14-23), 20 (16-24), and 8 (5-13) degrees, respectively. Most feet had some degree of varus (median 5 
degrees, $\left.\mathrm{P}_{25}-\mathrm{P}_{75}, 0-14\right)$. The original calcaneal fractures were classified according to Stephens as 2 type 1, 29 type 2, 3 type 3, and 17 unknown). Post-operatively the talocalcaneal, calcaneal inclination, and talar declination angles were 19 (14-25), 21 (18-25), and 8 (4-12) degrees, respectively. Most feet were in the neutral position, except for three that were still in 5 degrees or more of varus. There were $24 \%$ missing values because of absence of radiographs or specific views. In Table 1, the pre- and postoperative data are grouped according to the final type of arthrodesis, subtalar, or triple. No statistically significant differences between the two groups were noted.

Clinical results and correlations with outcome

Patients in both groups were comparable for sex, age at fracture, initial treatment, time to arthrodesis, follow-up, and post-operative radiological angles (Table 1). There were no statistically significant differences in outcome, as measured by the MFS, SF-36, and VAS ( $P=0.79,0.67$, and 0.21 , respectively; see Table 1 ).

In the group of responders with a non-union $(N=9)$ seven patients were smokers (78\%), whereas only 5 out of 19 patients without a non-union (26\%) were smokers $(P=0.017)$. In the entire group of patients $(N=56$ patients, with 61 arthrodeses), the correlation between smoking and the occurrence of a non-union was also significant $(P=0.021)$.

\section{Discussion}

The aim of this study was to compare the functional outcomes of an in situ subtalar or triple arthrodesis for the painful sequelae of an intra-articular calcaneal fracture, treated initially either conservatively or by means of osteosynthesis (ORIF or by percutaneous fixation using screws or K-wires) with a minimum follow-up of 2 years. No apparent statistically significant correlation between the type of arthrodesis and functional outcome was found, using a disease-specific outcome score (MFS), a generic quality of life scale (SF-36), and patient satisfaction (VAS) as outcome measures. The overall results of treatment in the questionnaires returned by thirty-three patients were fair (56 points on the MFS). Separation of the SF-36 results into the physical health and mental health components clearly showed that the physical part of the SF-36 was affected far more than the mental component by deviating from the norm-based score of 50 [24]. Patients experience significant difficulty from a previously fractured calcaneus.

Table 1 Data of patients with a subtalar or triple arthrodesis at follow-up

\begin{tabular}{|c|c|c|c|c|}
\hline Parameters & Total & Subtalar & Triple & $P$ \\
\hline Patients/feet $(n)$ & $33 / 37$ & $16 / 17$ & $17 / 20$ & - \\
\hline Male $(n)$ & 23 & 10 & 13 & $0.46^{\mathrm{b}}$ \\
\hline Median age at fracture (year) ${ }^{\mathrm{a}}$ & $39(32-45)$ & $43(31-54)$ & $36(32-44)$ & $0.44^{\mathrm{c}}$ \\
\hline Initial treatment $(\mathrm{C} / \mathrm{O} / \mathrm{P})$ & $24 / 6 / 7$ & $10 / 3 / 4$ & $14 / 3 / 3$ & $0.48^{\mathrm{b}}$ \\
\hline Time to arthrodesis (months) ${ }^{\mathrm{a}}$ & $29(19-55)$ & $27(12-33)$ & $42(23-62)$ & $0.69^{\mathrm{c}}$ \\
\hline Median follow-up (months) ${ }^{\mathrm{a}}$ & $116(52-156)$ & $110(45-146)$ & $127(80-159)$ & $0.16^{\mathrm{c}}$ \\
\hline \multicolumn{5}{|l|}{ Post-arthrodesis angles (degree) ${ }^{\mathrm{a}}$} \\
\hline Talocalcaneal & $20(16-27)$ & $20(14-28)$ & $20(16-26)$ & $0.85^{\mathrm{c}}$ \\
\hline Calcaneal inclination & $21(18-24)$ & $22(18-26)$ & $20(18-24)$ & $0.69^{\mathrm{c}}$ \\
\hline Talar declination & $8(6-12)$ & $9(7-13)$ & $8(6-11)$ & $0.58^{\mathrm{c}}$ \\
\hline Varus/valgus & $0(0-3)$ & $0(0-4)$ & $0(-4-2)$ & $0.29^{\mathrm{c}}$ \\
\hline Missing values (\%) & 24 & 21 & 25 & $0.43^{\mathrm{b}}$ \\
\hline Post-arthrodesis $\mathrm{BMI}^{\mathrm{a}}$ & $27.1(25.6-29.3)$ & $27.4(24.6-28.3)$ & $27.1(25.7-31.1)$ & $0.72^{\mathrm{c}}$ \\
\hline \multicolumn{5}{|l|}{ Outcome scores ${ }^{\mathrm{a}}$} \\
\hline MFS & $56(44-70)$ & $59(39-77)$ & $56(46-69)$ & $0.79^{\mathrm{c}}$ \\
\hline SF-36 (Total) & $83(72-97)$ & 84 (69-99) & $83(72-93)$ & $0.67^{\mathrm{c}}$ \\
\hline SF-36 (PSC) & $33(26-46)$ & $33(26-47)$ & $33(25-42)$ & $0.56^{\mathrm{c}}$ \\
\hline SF-36 (MSC) & $52(39-56)$ & $52(38-57)$ & $52(39-56)$ & $0.97^{\mathrm{C}}$ \\
\hline Post-VAS & $6(4-7)$ & $5(4-8)$ & $6(5-7)$ & $0.21^{\mathrm{c}}$ \\
\hline
\end{tabular}

$C$ initial conservative treatment, $O$ initial treatment using ORIF, $P$ initial percutaneous treatment, $B M I$ body mass index, $M F S$ Maryland foot score, $P S C$ physical score component, $M S C$ mental score component, VAS visual analogue scale

${ }^{\text {a }}$ Data are presented as median with the $\mathrm{P}_{25}$ and $\mathrm{P}_{75}$ between brackets

Data were analysed using the ${ }^{\mathrm{b}} \chi^{2}$ and ${ }^{\mathrm{c}}$ Mann-Whitney U-test. $P$-values below 0.05 were considered statistically significant 
Table 2 Overview of the literature of in situ subtalar arthrodesis

\begin{tabular}{|c|c|c|c|c|c|c|}
\hline Author (year) & $\begin{array}{l}\text { Patients }(n) \\
\text { [calcaneal \#] }\end{array}$ & $\begin{array}{l}\text { Average time between } \\
\text { fracture and arthrodesis (range) }\end{array}$ & $\begin{array}{l}\text { Follow-up since } \\
\text { arthrodesis (range) }\end{array}$ & $\begin{array}{l}\text { Union } \\
\text { rate }(\%)\end{array}$ & $\begin{array}{l}\text { AOFAS/other scoring } \\
\text { system (range) }\end{array}$ & $\begin{array}{l}\text { Return to } \\
\text { work }(\%)\end{array}$ \\
\hline Conn (1935) [39] & 39 [39] & - & - & - & $50-66 \% \mathrm{G}$ & - \\
\hline Kalamchi (1977) [40] & $6[6]$ & Range $6-12 \mathrm{~m}$ & Range $1.5-3.5 y$ & 100 & $100 \% \mathrm{~S}$ & 50 \\
\hline Johansson (1982) [38] & $21[23]$ & av $2.2 \mathrm{y}(1-5)$ & $4.3 y(2-9)$ & 100 & $96 \% \mathrm{G}+\mathrm{S}$ & 95 \\
\hline Russotti (1988) [41] & $45[17]$ & $28 \mathrm{~m}$ & $48 \mathrm{~m}$ & 98 & $71 \% \mathrm{~S}$ & 93 \\
\hline Mann (1988) [42] & 9 [5] & - & $42 \mathrm{~m} \mathrm{(23-103)}$ & 100 & $89 \% \mathrm{G}+\mathrm{E}$ & - \\
\hline Myerson (1993) [43] & $15[15]$ & $22 \mathrm{~m}(10-49)$ & $31 \mathrm{~m}(26-41)$ & 100 & $77(45-91)$ & 87 \\
\hline Stephens (1996) [21] & 19 [19] & $35 \mathrm{~m}(2-194)$ & $31 \mathrm{~m}(12-54)$ & 100 & $86 *(84 \% \mathrm{G}+\mathrm{E})$ & - \\
\hline Dahm (1998) [18] & 24 [19] & $4 y(0.3-33)$ & $4 y(2-6)$ & 96 & $68 \% \mathrm{G}+\mathrm{E}$ & 88 \\
\hline Mann (1998) [17] & 44 [12] & $3.5 y(1-13)$ & $60 \mathrm{~m} \mathrm{(24-177)}$ & 100 & 83 & - \\
\hline Sammarco (1998) [16] & $45[13]$ & - & $22.6 \mathrm{~m}$ & 93 & $82 \% \mathrm{~S}$ & - \\
\hline Chandler (1999) [32] & 18 [19] & $32 \mathrm{~m}(8-156)$ & $27 \mathrm{~m}(12-62)$ & 100 & 71 & 67 \\
\hline Thermann (1999) [5] & $40[40]$ & $3.5 y(0.25-20)$ & $5.2 \mathrm{y}(2-11)$ & 98 & $65 / 69 * *$ & - \\
\hline Easley (2000) [25] & $152[?]$ & $17 \mathrm{~m}(4-126)$ & $51 \mathrm{~m} \mathrm{(24-130)}$ & 85 & 70 & - \\
\hline Flemister (2000) [44] & 49 [49] & $28 \mathrm{~m}$ & $50 \mathrm{~m}$ & 96 & 75 & $61-71$ \\
\hline Kolodziej (2001) [45] & 9 [9] & $25 \mathrm{~m}(6-60)$ & 37 m 12-56) & 100 & $63(39-94)$ & 83 \\
\hline Savva (2007) [28] & $17[17]$ & $41 \mathrm{~m}(7-288)$ & 79 m (48-94) & - & $78(48-94)$ & 94 \\
\hline Davies (2007) [15] & $96[ \pm 67]$ & - & - & 94 & $71 \% \mathrm{G} * * *$ & - \\
\hline Radnay (2009) [34] & 69 & $23 \mathrm{~m}$ & $63 \mathrm{~m}$ & 92 & $74 / 87 * *$ & - \\
\hline
\end{tabular}

This table shows the results from several studies concerning in situ subtalar arthrodesis. The number of patients and the number of calcaneal fractures are given, the time between the injury and the arthrodesis, and the duration of follow-up with the main findings

$E$ excellent, $G$ good, $S$ satisfactory, $y$ years, $m$ months

$*$ MFS, ** scores for conservatively and operatively treated patients respectively, *** Angus-Cowell score

A secondary triple arthrodesis after an intra-articular calcaneal fracture has not been described frequently in the literature. Over 500 cases of in situ subtalar arthrodesis performed after failed treatment of intra-articular calcaneal fractures were identified in numerous studies (Table 2), in contrast only 14 cases of secondary triple arthrodesis in three studies were found as a salvage technique after calcaneal fractures (Table 3).

The results in this study compare negatively to the literature as summarised in Table 2. One study applying the MFS found an average score of 86 in 19 patients; [21] and with the use of the American Orthopaedic Foot and Ankle Society Hindfoot score (maximum of 94 points), an outcome score between 63 and 83 points has previously been found. All other studies classify patients into excellent, good, or satisfactory groups, making comparisons slightly more difficult. Some studies reporting on the results of arthrodesis present mixed populations with acquired flat feet (e.g. tibial tendon dysfunction), subtalar dislocations, non-traumatic arthritis, and as sequelae of calcaneal fractures. The results of arthrodesis after calcaneal fractures have been reported to be inferior compared to arthrodesis for other causes [17, 25].

The fact that the results were only fair might be attributed to several factors. A rather high rate of non-union was found in this study, which might be partially explained by the high percentage of patients that smoked and a relatively high rate of revision surgeries for previously

Table 3 Overview of the literature of secondary in situ triple arthrodesis

\begin{tabular}{lllllll}
\hline Author (year) & $\begin{array}{l}\text { Patients }(n) \\
\text { [calcaneal \#] }\end{array}$ & $\begin{array}{l}\text { Average time between fracture } \\
\text { and arthrodesis (range) }\end{array}$ & $\begin{array}{l}\text { Follow-up since } \\
\text { arthrodesis (range) }\end{array}$ & $\begin{array}{l}\text { Union } \\
\text { rate }(\%)\end{array}$ & $\begin{array}{l}\text { AOFAS/other scoring } \\
\text { system (range) }\end{array}$ & $\begin{array}{l}\text { Return to } \\
\text { work }(\%)\end{array}$ \\
\hline Conn (1935) [39] & $6[6]$ & - & - & - & $83 \% \mathrm{G}$ \\
Myerson (1993) [43] & $5[5]$ & $33 \mathrm{~m} \mathrm{(19-50)}$ & $34 \mathrm{~m} \mathrm{(26-44)}$ & - & $51(40-65)^{*}$ \\
Kolodziej (2001) [45] & $3[3]$ & $18 \mathrm{~m} \mathrm{(5-36)}$ & $88 \mathrm{~m} \mathrm{(63-112)}$ & 100 & $72(50-94)$ & 80 \\
\hline
\end{tabular}

$E$ excellent, $G$ good, $S$ satisfactory, $y$ years, $m$ months. * Modified AOFAS

This table shows the results from several studies concerning in situ triple arthrodesis. The number of patients and the number of calcaneal fractures are given, the time between the injury and the arthrodesis, and the duration of follow-up with the main findings 
failed subtalar arthrodeses. A correlation between impaired healing of the arthrodesis and smoking has been demonstrated in other studies [25-27]; there is also a relationship between delayed healing and revision surgery, [25] and with the amount of avascular subchondral bone at the subtalar joint [25]. At our institute, no per-operative data exist concerning the condition of the subtalar joint during the procedure.

There might have been a negative selection bias, in which more patients with persistent complaints returned the questionnaire. In addition, particular details of the operative technique might have been crucial in some cases: for example, resection of the lateral wall [17, 20, 21, 28-32] was not performed routinely at our institution $(N=2)$. There was no delineated treatment protocol [30], and different methods of bone grafting and fixation have been used in the current study. To what extent these factors contributed to less favourable results remains speculative.

There is discussion whether the initial treatment modality influences the functional outcome after an arthrodesis. Regardless of the type of arthrodesis, current data revealed no statistically significant difference in the overall median MFS, VAS, and SF-36 after initial conservative treatment $(55,6$, and 83 , respectively) versus initial operative treatment $(64,5$, and 87 , respectively; data not shown). However, statistical power was insufficient to make a definitive statement. Thermann found an AOFAS score of 69 points in the initially operated group $(N=17)$ vs. 65 in the conservative group $(N=23)$, suggesting a trend towards improved functional outcome after subtalar arthrodesis included in the initial operative regime [5]. Others found no correlation with the initial treatment and the arthrodesis at follow-up [32, 33]. A recent study showed the clear benefit of initial operative treatment versus conservative treatment after a subtalar arthrodesis using the Maryland Foot Score and the American Orthopaedic Foot Ankle Society Hindfoot score [34].

The $24 \%$ missing values in the radiological evaluation was due to a difference in the follow-up protocol for the trauma and orthopaedic surgeons. A lateral image of the hindfoot was made in all cases; an anteroposterior image of the ankle was made by the orthopaedic surgeons and an axial image of the calcaneus by the trauma surgeons only.

Several authors have pointed out the importance of performing a triple arthrodesis after failed treatment of displaced intra-articular calcaneal fractures, e.g. Conn [35], Harris [36], and Bankart [37]. And the need for a comparative study of subtalar versus triple arthrodesis had been emphasised by Jahss in 1982 [38]. Despite the limitations of this study, it is the first to compare in situ subtalar and triple arthrodesis after an intra-articular calcaneal fracture. The data of the current study suggest that there is no statistically significant difference in functional outcome between secondary in situ subtalar or triple arthrodesis as therapy for symptomatic arthrosis after an intra-articular calcaneal fracture. Moreover, current data confirmed that smoking is a risk factor for non-union, as shown previously [25-27]. A standardised treatment protocol (including the surgical technique and follow-up strategy) might improve the outcome and would enable the comparison of different techniques.

Conflict of interest None.

\section{References}

1. Schepers T, van Lieshout EM, van Ginhoven TM, Heetveld MJ, Patka P (2008) Current concepts in the treatment of intra-articular calcaneal fractures: results of a nationwide survey. Int Orthop 32(5):711-715

2. Buckley R, Tough S, McCormack R, Pate G, Leighton R, Petrie $\mathrm{D}$ et al (2002) Operative compared with nonoperative treatment of displaced intra-articular calcaneal fractures: a prospective, randomized, controlled multicenter trial. J Bone Joint Surg Am 84-A(10): 1733-1744

3. Crosby LA, Fitzgibbons T (1993) Intraarticular calcaneal fractures. Results of closed treatment. Clin Orthop 290:47-54

4. Zwipp H, Rammelt S, Barthel S (2004) Calcaneal fracturesopen reduction and internal fixation (ORIF). Injury 35(Suppl 2): SB46-SB54

5. Thermann H, Hufner T, Schratt E, Held C, von Glinski S, Tscherne H (1999) Long-term results of subtalar fusions after operative versus nonoperative treatment of os calcis fractures. Foot Ankle Int 20(7):408-416

6. Burton DC, Olney BW, Horton GA (1998) Late results of subtalar distraction fusion. Foot Ankle Int 19(4):197-202

7. Carr JB, Hansen ST, Benirschke SK (1988) Subtalar distraction bone block fusion for late complications of os calcis fractures. Foot Ankle 9(2):81-86

8. James ET, Hunter GA (1983) The dilemma of painful old os calcis fractures. Clin Orthop 177:112-115

9. Angus PD, Cowell HR (1986) Triple arthrodesis. A critical longterm review. J Bone Joint Surg Br 68(2):260-265

10. Howorth MB (1974) Triple subtalar arthrodesis. Clin Orthop Relat Res 99:175-180

11. Miller RA, Hayes W (1994) Subtalar arthrodesis with screw fixation in de adult. Oper Tech Orthop 4(4):169-172

12. Wilcox DK, Anderson JG (2001) Advances in the surgical treatment of ankle and hindfoot arthritis. Curr Opin Orthop 12:87-92

13. Zwipp H, Tscherne H, Thermann H, Weber T (1993) Osteosynthesis of displaced intraarticular fractures of the calcaneus. Results in 123 cases. Clin Orthop 290:76-86

14. Schepers T, Schipper IB, Vogels LM, Ginai AZ, Mulder PG, Heetveld MJ et al (2007) Percutaneous treatment of displaced intra-articular calcaneal fractures. J Orthop Sci 12(1):22-27

15. Davies MB, Rosenfeld PF, Stavrou P, Saxby TS (2007) A comprehensive review of subtalar arthrodesis. Foot Ankle Int 28(3):295-297

16. Sammarco GJ, Tablante EB (1998) Subtalar arthrodesis. Clin Orthop Relat Res 349:73-80

17. Mann RA, Beaman DN, Horton GA (1998) Isolated subtalar arthrodesis. Foot Ankle Int 19(8):511-519 
18. Dahm DL, Kitaoka HB (1998) Subtalar arthrodesis with internal compression for post-traumatic arthritis. J Bone Joint Surg Br 80(1):134-138

19. Schepers T, Ginai AZ, Mulder PG, Patka P (2007) Radiographic evaluation of calcaneal fractures: to measure or not to measure. Skeletal Radiol 36(9):847-852

20. Paley D, Hall H (1993) Intra-articular fractures of the calcaneus. A critical analysis of results and prognostic factors. J Bone Joint Surg Am 75(3):342-354

21. Stephens HM, Sanders R (1996) Calcaneal malunions: results of a prognostic computed tomography classification system. Foot Ankle Int 17(7):395-401

22. Sanders R, Fortin P, DiPasquale T, Walling A (1993) Operative treatment in 120 displaced intraarticular calcaneal fractures. Results using a prognostic computed tomography scan classification. Clin Orthop 290:87-95

23. Schepers T, Heetveld MJ, Mulder PG, Patka P (2008) Clinical outcome scoring of intra-articular calcaneal fractures. J Foot Ankle Surg 47(3):213-218

24. Ware JE Jr (2000) SF-36 health survey update. Spine 25(24): 3130-3139

25. Easley ME, Trnka HJ, Schon LC, Myerson MS (2000) Isolated subtalar arthrodesis. J Bone Joint Surg Am 82(5):613-624

26. Chahal J, Stephen D, Bulmer B, Daniels T, Kreder H (2006) Factors associated with outcome after subtalar arthrodesis. J Orthop Trauma 20(8):555-561

27. Bednarz PA, Beals TC, Manoli A II (1997) Subtalar distraction bone block fusion: an assessment of outcome. Foot Ankle Int 18(12):785-791

28. Savva N, Saxby TS (2007) In situ arthrodesis with lateral-wall ostectomy for the sequelae of fracture of the os calcis. J Bone Joint Surg Br 89(7):919-924

29. Cabot H, Binney H (1907) Fractures of the os calcis and astragalus. Ann Surg 45(1):51-68

30. Clare MP, Lee WE III, Sanders RW (2005) Intermediate to longterm results of a treatment protocol for calcaneal fracture malunions. J Bone Joint Surg Am 87(5):963-973
31. Cotton FJ (1921) Old os calcis fractures. Ann Surg 74(3):294303

32. Chandler JT, Bonar SK, Anderson RB, Davis WH (1999) Results of in situ subtalar arthrodesis for late sequelae of calcaneus fractures. Foot Ankle Int 20(1):18-24

33. Rammelt S, Grass R, Zawadski T, Biewener A, Zwipp H (2004) Foot function after subtalar distraction bone-block arthrodesis. A prospective study. J Bone Joint Surg Br 86(5):659-668

34. Radnay CS, Clare MP, Sanders RW (2009) Subtalar fusion after displaced intra-articular calcaneal fractures: does initial operative treatment matter? J Bone Joint Surg Am 91(3):541-546

35. Gallie WE (1943) Subastragalar arthrodesis in fractures of the os calcis. J Bone Joint Surg 25:731-736

36. Harris RI (1946) Fractures of the os calcis; their treatment by triradiate traction and subastragalar fusion. Ann Surg 124:1082-1100

37. Bankart ASB (1942) Fractures of the os calcis. Lancet 240(6207): 175

38. Johansson JE, Harrison J, Greenwood FA (1982) Subtalar arthrodesis for adult traumatic arthritis. Foot Ankle 2(5):294-298

39. Conn HR (1935) The treatment of fractures of the os calcis. J Bone Joint Surg Am 17(2):392-405

40. Kalamchi A, Evans JG (1977) Posterior subtalar fusion. A preliminary report on a modified Gallie's procedure. J Bone Joint Surg Br 59(3):287-289

41. Russotti GM, Cass JR, Johnson KA (1988) Isolated talocalcaneal arthrodesis. A technique using moldable bone graft. J Bone Joint Surg Am 70(10):1472-1478

42. Mann RA, Baumgarten M (1988) Subtalar fusion for isolated subtalar disorders. Preliminary report. Clin Orthop Relat Res 226:260-265

43. Myerson M, Quill GE Jr (1993) Late complications of fractures of the calcaneus. J Bone Joint Surg Am 75(3):331-341

44. Flemister AS Jr, Infante AF, Sanders RW, Walling AK (2000) Subtalar arthrodesis for complications of intra-articular calcaneal fractures. Foot Ankle Int 21(5):392-399

45. Kolodziej P, Nunley JA (2001) Outcome of subtalar arthrodesis after calcaneal fracture. J South Orthop Assoc 10(3):129-139 